\title{
MicroRNA-193b acts as a tumor suppressor gene in human esophageal squamous cell carcinoma via target regulation of KRAS
}

\author{
MIN KANG ${ }^{1,2}$, YAN LI $^{3}$, SHENGTAO ZHU ${ }^{1}$, SHUTIAN ZHANG ${ }^{1}$, SHUILONG GUO $^{1}$ and PENG LI ${ }^{1}$ \\ ${ }^{1}$ Department of Gastroenterology, Beijing Friendship Hospital, Capital Medical University, National Clinical Research \\ Center for Digestive Diseases, Beijing Digestive Disease Center, Beijing Key Laboratory for Precancerous Lesion of \\ Digestive Diseases, Beijing 100050; ${ }^{2}$ Department of Digestive Diseases; ${ }^{3}$ Molecular Medicine Experimental Center, \\ Affiliated Hospital of Southwest Medical University, Luzhou, Sichuan 646000, P.R. China
}

Received March 29, 2018; Accepted November 12, 2018

DOI: $10.3892 / \mathrm{ol} .2019 .10039$

\begin{abstract}
In recent years, microRNA-193b (miR-193b) is regarded as a tumor suppressor in the development and progression of various cancers. Several studies have indicated that KRAS could be regulated by miR-193b in pancreatic cancer cells. However, the function of miR-193b in human esophageal squamous cell carcinoma has not been explored intensively thus far. Herein, the relationship between miR-193b and KRAS was mainly explored in esophageal squamous cell carcinoma cells. In the present study, the expression levels of miR-193b and KRAS were assessed in both human esophageal cancer cells and tissues. The direct regulatory relationship between miR-193b and KRAS was evaluated using dual-luciferase assay. The effect of miR-193b overexpression and inhibitor on cell proliferation, migration/invasion, and apoptosis was further detected herein. Our results indicated that the expression of miR-193b was significantly lower in human esophageal cancer tissues than paracancerous tissues. The expression level of miR-193b/KRAS was stage-dependent in human esophageal cancers. KRAS was indicated as the direct target of miR-193b, and upregulation of miR-193b increased the percentage of cell apoptosis, and suppressed cell proliferation as well as cell migration/invasion via direct regulation of KRAS. Therefore, our study indicated that miR-193b
\end{abstract}

Correspondence to: Dr Shuilong Guo or Dr Peng Li, Department of Gastroenterology, Beijing Friendship Hospital, Capital Medical University, National Clinical Research Center for Digestive Diseases, Beijing Digestive Disease Center, Beijing Key Laboratory for Precancerous Lesion of Digestive Diseases, 95 Yong'an Road, Xicheng, Beijing 100050, P.R. China

E-mail: slong.guo@163.com

E-mail: lipeng@ccmu.edu.cn

Key words: miR-193b, KRAS, esophageal squamous cell carcinoma, cell viability, cell apoptosis plays an important role in the development and progression of human esophageal squamous cell carcinoma, which may become a novel target in the treatment of human esophageal squamous cell carcinoma in the future.

\section{Introduction}

Esophageal cancer is considered as the 7 th most common type of cancer worldwide. It is also the 6th leading cause of cancer-associated mortality $(1,2)$. According to different histological types, esophageal cancer can be further divided into squamous cell carcinoma and adenocarcinoma. Squamous cell carcinoma is regarded as the most prevalent esophageal cancer in the world, especially in some developing countries (3). Although some advances has been made in diagnosis and treatments, the incidence of esophageal cancer has markedly increased in the past years (3), and the 5-year survival rate of patients with advanced esophageal cancer is less than $20 \%$ (4). Therefore, the development of more effective therapeutic methods and novel prognostic molecular markers are necessary to improve the patient survival rate.

Numerous microRNAs (miRNAs or miRs) have been demonstrated to act as oncogenes or tumor suppressor genes. During the process of cancer development and progression, miRNAs have been revealed to play a significant role in regulation. In addition, these miRNAs also can be used as novel molecular biomarkers for cancer prognosis, even cancer targeted therapies (5-8). Among these miRNAs, researchers have demonstrated that microRNA-193b (miR-193b) functions as a tumor suppressor in multiple malignancies, such as prostate cancer (9), melanoma (10), and breast cancer (11). In addition, the level of miR-193b was revealed to be different between chemosensitive and chemoresistant cell lines, and the chemoresistant cell lines had lower expression than chemosensitive cell lines (12). The overexpression of miR-193b was revealed to significantly impede the ability of esophageal cancer cells to recover following 5-fluorouracil treatment, and markedly induce autophagic flux and non-apoptotic cell death, indicating the important role of miR-193b in esophageal cancer chemotherapy $(12,13)$. 
Molecular alterations associated with esophageal cancer progression have been extensively investigated in recent years $(3,14)$. One of the most frequent molecular alterations is KRAS, an oncogene. Activated KRAS could cause cell growth and survival, which is important during cancer development $(15,16)$. Although KRAS mutations are regarded as a key event in carcinogenesis, the targeting upstream signaling which modulates KRAS activity is still a promising future approach for the treatment of esophageal cancer.

In present study, KRAS was confirmed as the direct target gene of miR-193b in human esophageal squamous cell carcinoma. It was also revealed that miR-193b overexpression could induce esophageal squamous cell carcinoma cell apoptosis and suppress cancer cell proliferation, as well as migration/invasion, indicating that miR-193b has the potential to become a novel diagnosis marker and therapy target for human esophageal squamous cell carcinoma.

\section{Materials and methods}

Esophageal squamous cell carcinoma samples. In our study, 53 different patients (38 males and 15 females) donated their esophageal squamous cell carcinoma tissues and paracancerous tissues for our research. All samples belonged to primary tumors. The cancer tissues from 31 patients were infiltrating esophageal squamous cell carcinoma and the cancer tissues from 22 patients were superficial esophageal squamous cell carcinoma. The number of patients in the different age-groups was as follows: $\mathrm{n}(50-59)=9 ; \mathrm{n}(60-69)=30 ; \mathrm{n}(70=79)=14$. The number of patients in the different cancer stages wasas follows: $\mathrm{n}($ Stage I $)=6 ; \mathrm{n}($ stage II $)=16 ; \mathrm{n}($ stage III $)=20 ; \mathrm{n}($ stage IV $)=11$.

Cell culture. Esophageal squamous cell carcinoma cell lines, KYSE450 and TE1, and normal epithelial cell line, Het-1A, were used in the present study, and were purchased from the Cell Bank of the Chinese Academy of Sciences (Shanghai, China). The cells were cultured using Dulbecco's Modified Eagle's Medium (DMEM; Hyclone; GE Healthcare Life Sciences, Logan, UT, USA) supplemented with $10 \%$ fetal bovine serum (FBS; Hyclone; GE Healthcare Life Sciences), $0.1 \mathrm{~g} / \mathrm{ml}$ streptomycin and $100 \mathrm{U} / \mathrm{ml}$ penicillin (Sigma-Aldrich; Merck KGaA, Darmstadt, Germany) in a humidified $37^{\circ} \mathrm{C}$ incubator with $5 \% \mathrm{CO}_{2}$. The culture medium was changed every two days, and the cells were passaged by 1:4 dilution every 5-6 days.

miR-193b preparation and transfection. miR-193b (5'-AAC UGGCCCUCAAAGUCCCGCU-3') was synthesized from Sengong Biotech, Shanghai, China. The unspecific miRNA (5'-ACGUGACACGUUCGGAGAAUU-3') was used as the negative control (ctrl miRNA). The reverse complementary miRNA (5'-AGCGGGACUUUGAGGCCAGUU-3') was applied as a miR-193b inhibitor in this study. Cell transfection was performed using Lipofectamine 3000 transfection reagent (Invitrogen; Thermo Fisher Scientific, Inc.) according to the manufacturer's instructions.

Real-Time quantitative PCR. The real-time quantitative PCR procedure was the same as previously described (17-19). In the present study, KRAS was normalized to $18 \mathrm{~S}$ rRNA and
miR-193b was normalized to U6. The primer sequences (5'-3') were as follows: KRAS forward, 5'-GCCTTGACGATACAG CTAAT-3' and reverse, 5'-GCTGTGTCGAGAATATCC AA-3'; 18S rRNA forward, 5'-CCTGGATACCGCAGCTAG GA-3' and reverse, 5'-GCGGCGCAATACGAATGCCCC-3'; miR-193b forward, 5'-ACACTCCAGCTGGGAACTGGC CCTCAAAGTC-3' and reverse, 5'-AGCCTCTGCGCACGT GTTC-3'; U6 forward, 5'-CTCGCTTCGGCAGCACA-3' and reverse, 5'-AACGCTTCACGAATTTGCGT-3'.

Dual luciferase assay. The potential target genes of miR-193b were analyzed using TargetScan (http://www.targetscan. org/vert_72/). The 3'UTR sequence of the human KRAS gene was amplified using PCR and further cloned into a psiCHECK-based luciferase plasmid (Addgene, Inc., Cambridge, MA, USA). The establishment of mutated psiCHECK-KRAS 3'UTR, as well as the procedure of the dual luciferase assay was same as previously described $(20,21)$. In brief, the WT psiCHECK-KRAS 3'UTR was used as the template for PCR, and the primers which matched the 3'UTR part and contained the mutated sequence were synthesized by IDT (Beijing, China). The extension of the PCR template could generate nicked circular DNA molecules, followed by DpnI endonuclease digestion, competent cell transformation and mini-plasmid preparation. Herein, 3-4 bases could be mutated using the above method to induce mutation in psiCHECK-KRAS 3'UTR at once. Therefore, to obtain the whole mutated sequence (11-base mutation), the mutation induction was repeated for 3 times with different primers. The sequence of primers (5'-3') were as follows: M1-F, GCTTGTGACATTAAAAGATTAAAACGG CCAGTTATAGCTTATTAGGTGTTGA and M1-R, TCA ACACCTAATAAGCTATAACTGGCCGTTTTAATCTTTT AATGTCACAAGC; M2-F, GCTTGTGACATTAAAAGA TTAAAACCCGGAGTTATAGCTTATTAGGTGTTGA and M2-R, TCAACACCTAATAAGCTATAACTCCGGGTTTTA ATCTTTTAATGTCACAAGC; M3-F, GCTTGTGACATT AAAAGATTAAAACCCGGTCATATAGCTTATTAGGTGTTG A and M3-R, TCAACACCTAATAAGCTATATGACCGG GTTTTAATCTTTTAATGTCACAAGC.

The dual luciferase assay was performed using a Dual Luciferase Assay Kit according to the manufacturer's instructions (Promega Corporation, Madison, WI, USA). In the present study, both Firefly and Renilla luciferase values were detected. In addition, for the evaluation of relative luciferase activity, the firefly value was used in Renilla value normalization.

Colony-forming ability assay and various other assays. To evaluate the colony-forming ability of ctrl miRNA group, miR-193b group and inhibitor group, 100 cancer cells were seeded into 12 -well plates and incubated for 7 days at $37^{\circ} \mathrm{C}$ in an incubator with $5 \% \mathrm{CO}_{2}$. Then the cancer cells were fixed with $75 \%$ ethanol. The plate was further stained using crystal violet for 20 min. Finally, an Epson Perfection V600, Epson, Japan scanner was used to scan the plate and the results were further analyzed using BioSpot ${ }^{\circledR}$ software 5.0, Cellular Technology Limited (CTL), USA.

Cell proliferation, cell cycle analysis, cell apoptosis, and cell migration/invasion assays were performed as previously described (22). 
Statistical analysis. In the present study, the results were expressed as the means \pm SEM, and analysis was performed using SPSS 17.0 software (SPSS, Inc., Chicago, IL, USA. Unpaired Student's t-tests were used to analyze the means of two groups. One-way ANOVA with Bonferroni's correction was used to analyze the means of three or more groups. $\mathrm{P}<0.05$ was considered to indicate a statistically significant difference. In Fig. 1A and B, the level of paracancerous tissue group was regarded as ' 1 '. In Fig. 2A and B, the level of the control group (Ctrl miRNA) was regarded as ' 1 '. In Fig. 2C, the level of the control group [(KRAS-3'-UTR(WT) and KRAS-3'-UTR(Mu)] was regarded as ' 1 '.

\section{Results}

KRAS is the direct target of miR-193b in esophageal cancer cells. In the present study, esophageal squamous cell carcinoma tissues and paracancerous tissues from 53 different patients were harvested and qPCR was used to evaluate the relationship between KRAS and miR-193b. The results revealed that the mRNA level of miR-193b was significantly higher in human paracancerous tissues than human esophageal cancer tissues $(\mathrm{P}<0.05$; Fig. 1B), while the miRNA level of KRAS revealed the opposite tendency in human esophageal cancer tissues and paracancerous tissues $(\mathrm{P}<0.05$; Fig. $1 \mathrm{~B})$, indicating that there may be a negative regulatory relationship between miR-193b and KRAS in the esophageal cancer tissues of patients. In addition, the correlation between KRAS and miR-193b expression was also confirmed through linear regression analysis, and the results indicated that the increased miR-193b expression was significantly correlated with decreased KRAS expression in both paracancerous tissues $(y=-1.255 x+2.2664$, $\mathrm{R}^{2}=0.930$; Fig. 1C) and cancer tissues $(\mathrm{y}=-0.1934 \mathrm{x}+1.0448$, $\mathrm{R}^{2}=0.817$; Fig. 1D). In addition, the relationship between miR-193b/KRAS expression and stage of cancers was further analyzed herein. The results revealed no obvious difference between stages I and II, while increased expression of KRAS and decreased expression of miR-193b could be observed in stages III and IV compared to stage I. Therefore, the expression level of miR-193b/KRAS was stage-dependent in human esophageal cancers, and may potentially be novel diagnosis and prognostic molecular markers (Fig. 1E and F).

In addition, two different esophageal squamous cell carcinoma cell lines, KYSE450 and TE1, were used to analyze the negative regulatory relationship between miR-193b and KRAS in esophageal cancer, and the level of KRAS and miR-193b in Het-1A, normal epithelial cell line, was also detected using qPCR. It was determined that the mRNA level of KRAS was inhibited by the upregulation of miR-193b in human esophageal squamous cell carcinoma cells. In addition, the cancer cells transfected with the miR-193b inhibitor revealed significantly higher levels of KRAS compared with the control group ( $\mathrm{P}<0.05$; Fig. 2A and B). Compared with normal epithelial cells, Het-1A, both KYSE450 and TE1 exhibited a significantly higher expression of KRAS and a lower expression of miR-193b ( $\mathrm{P}<0.05$, Fig. 2C and D).

The potential target genes of miR-193b were analyzed using TargetScan, indicating that miR-193b could target the 3'UTR of KRAS and regulate this gene directly (the binding relationship was revealed in Fig. 2E). Therefore, both wild-type
(WT) and mutant-type (Mu) 3'UTR of KRAS were cloned into the psi-CHECK vector, followed by TE1 cell transfection and dual-luciferase assay. The results revealed that miR-193b significantly suppressed the luciferase activity in the WT KRAS-3'-UTR group, but did not reveal a significant effect in the Mu KRAS-3'-UTR group ( $\mathrm{P}<0.05$; Fig. $2 \mathrm{E})$. In addition, the inhibitory effect of miR-193b was reversed by the transfection with the miR-193b inhibitor in the WT KRAS-3'-UTR group, but no significant difference was observed in the Mu KRAS-3'-UTR group $(\mathrm{P}<0.05$; Fig. 2E). Collectively, these assays revealed the direct targeted regulation between KRAS and miR-193b.

miR-193b inhibits the growth and proliferation abilities of esophageal cancer cell lines. The difference in cell proliferation ability among the control miRNA transfection group, the miR-193b overexpression group and the miR-193b inhibitor group was analyzed in the present study. The CCK-8 results revealed that esophageal cancer cell proliferation ability was enhanced via miR-193b inhibitor transfection. In addition, the overexpression of miR-193b significantly suppressed the cell proliferation ability compared to the control cells (Fig. 3A). A rescue experiment was also performed, and KRAS overexpression was revealed to significantly increase the proliferation ability of esophageal cancer cells. miR-193b overexpression inhibited the promoting effect of KRAS overexpression, indicating the effect of miR-193b on cell viability via regulation of KRAS (Fig. 3B).

Furthermore, the proliferation ability of different groups was further detected via colony formation assay. The results revealed that the colony formation ability was increased in the miR-193b-inhibitor transfection group. miR-193b overexpression significantly decreased the number of colonies in esophageal squamous cell carcinoma cells, which was consistent with the CCK-8 results (Fig. 3C).

In addition, the regulation of miR-193b and KRAS on the cell cycle was assessed by flow cytometric assay. The results revealed that miR-193b overexpression increased the percentage of cells at the G0/G1 phase and decreased the percentage of S-phase and G2/M-phase cells of KYSE450 and TE1. The opposite phenomenon was revealed in the miR-193b inhibitor group ( $\mathrm{P}<0.05$, Fig. 3D). Collectively, these results indicated that miR-193b inhibited the proliferation ability of esophageal cancer cell lines.

Effect of miR-193b on esophageal cancer cell apoptosis. In our study, cell apoptosis was assessed using Annexin V/PI staining. Both early-stage apoptotic cells (Annexin V-positive and PI-negative) and late-stage apoptotic cells (Annexin V-positive and PI-positive) were analyzed. In the KYSE450 cells, it was revealed that the percentage of early-stage apoptotic cells was significantly increased in the miR-193b overexpression group, while transfection with the miR-193b inhibitor significantly decreased the level of both early-stage and late-stage apoptotic cells compared with the control group. In the TE1 cells, both the percentages of early-stage and late-stage apoptotic cells were significantly increased with the overexpression of miR-193b, however, miR-193b-inhibitor transfection only reduced the percentage of late-stage apoptotic cells (Fig. 4A and B), indicating that miR-193b regulated cell apoptosis of esophageal cancer cells. 
A
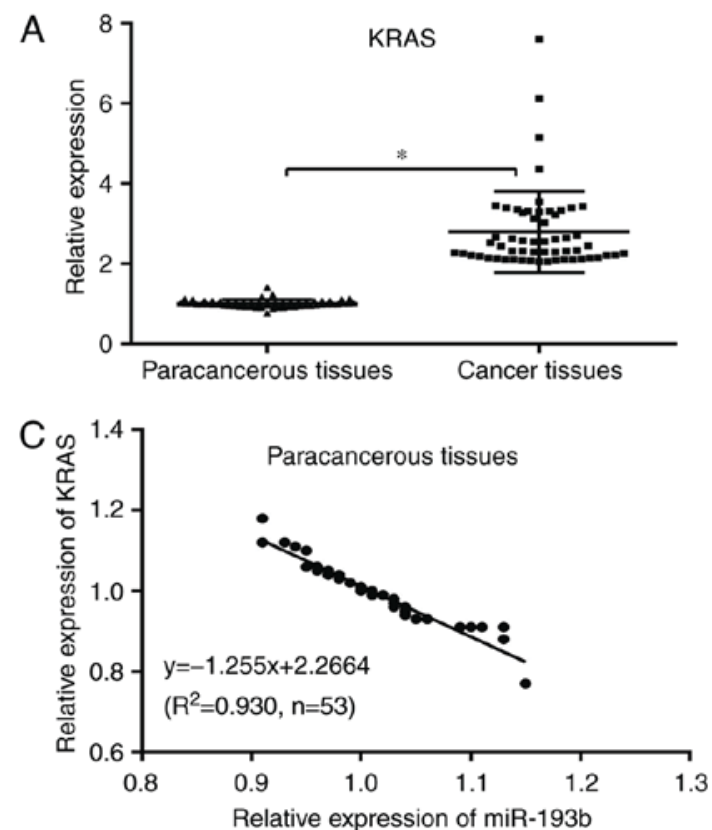

$\mathrm{E}$

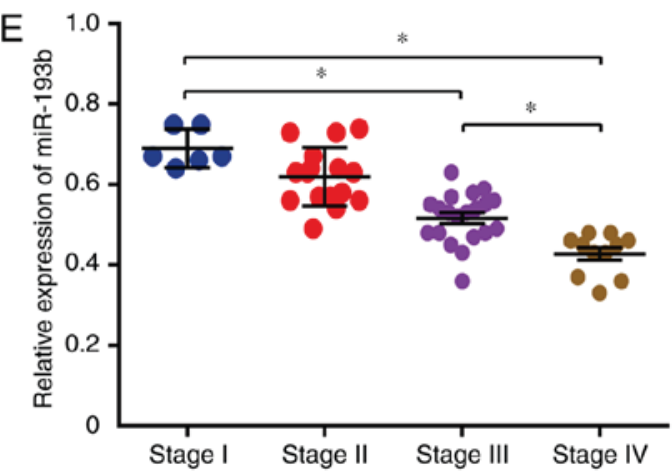

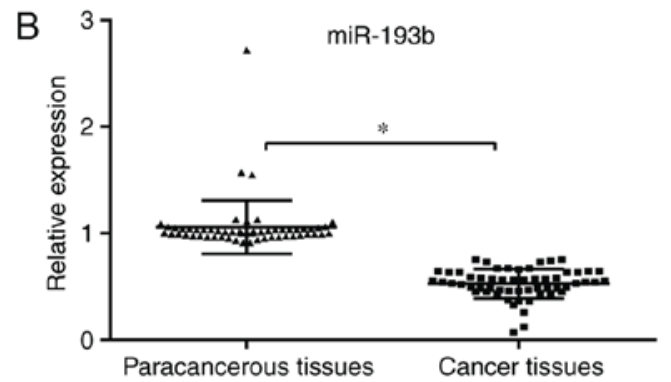
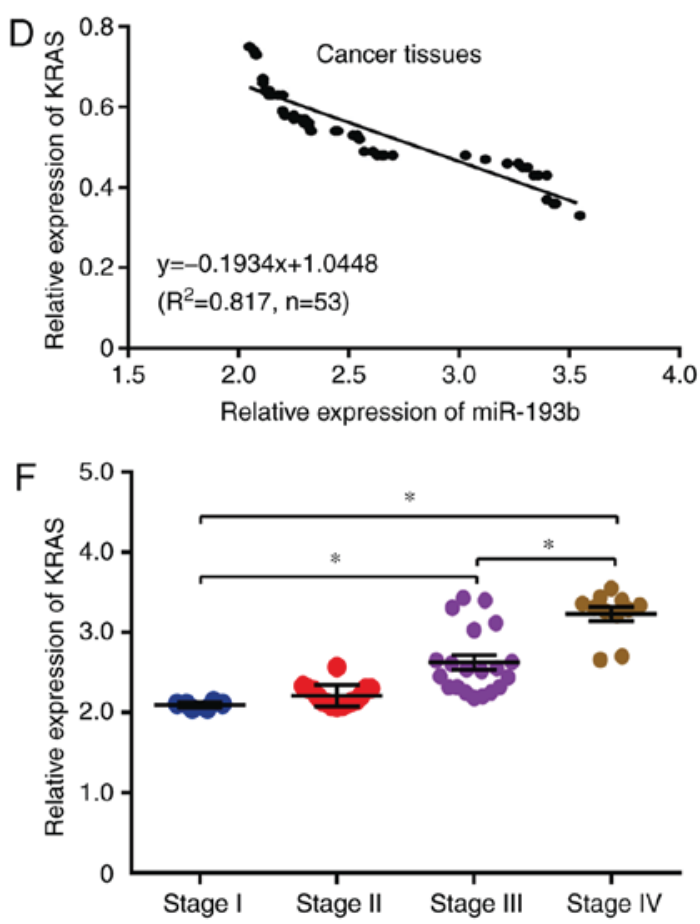

Figure 1. Expression of miR-193b and KRAS in human esophageal squamous cell carcinoma tissues and paracancerous tissues. (A and B) The expression of (A) KRAS and (B) miR-193b was assessed by qPCR respectively. (C and D) Linear regression analysis between miR-193b and KRAS in human esophageal cancer tissues and paracancerous tissues. (E and F) Expression level of miR-193b and KRAS in different cancer stages. "P $<0.05$ between the two groups. miR-193b, microRNA-193b.

miR-193b inhibits esophageal cancer cell migration and invasion. The effect of miR-193b on cell migration and invasion abilities was further analyzed in the present study. In TE1 cancer cells, the results indicated that both cell migration and invasion could significantly be enhanced via miR-193b-inhibitor transfection. Compared with the control group, the migration and invasion abilities of human esophageal cancer cells were suppressed in the miR-193b overexpression group, indicating the key role of miR-193b in the migration/invasion of human esophageal cancer cells ( $\mathrm{P}<0.05$; Fig. 5A and B). A similar tendency was revealed in the results of KYSE450 cells, however, in this cell line, miR-193b-inhibitor transfection did not significantly affect cell invasion ability.

\section{Discussion}

The key role of miR-193b in the progression and development of various cancers has been revealed in studies $(10,23-25)$. In recent years, researchers revealed that miR-193b could promote autophagy as well as non-apoptotic cell death in esophageal cancer cells (13). In addition, although critical mRNA targets of miR-193b were unknown, both target prediction and siRNA data analysis indicated that miR-193b may mediate these effects through stathmin 1 regulation (13). Researchers also revealed that the silencing of stathmin 1 could at least partially reverse the enhanced sensitivity to 5-fluorouracil, similar to the results obtained with overexpression of miR-193b, and miR-193b was revealed to inhibit tumor growth and metastasis, consequently through regulation of the expression of stathmin 1 in other cancer cells (26). Therefore, these data revealed the important role of miR-193b in the regulation of esophageal cancer progression. In addition, a previous study revealed the target relationship between miR-193b and KRAS in pancreatic cancer (24). However, whether miR-193b can negatively regulate the expression of KRAS, and whether it is related to clinical and pathological features in human esophageal cancer, has not been investigated intensively. In the present study, it was demonstrated that miR-193b targeted the 3'UTR of KRAS, and regulated the expression of KRAS negatively in both human esophageal squamous cell carcinoma tissues and cells. The expression level of miR-193b/KRAS was stage-dependent in human esophageal cancer. miR-193b overexpression promoted cell apoptosis and significantly inhibited the proliferation and migration/invasion abilities of esophageal squamous cell 
A

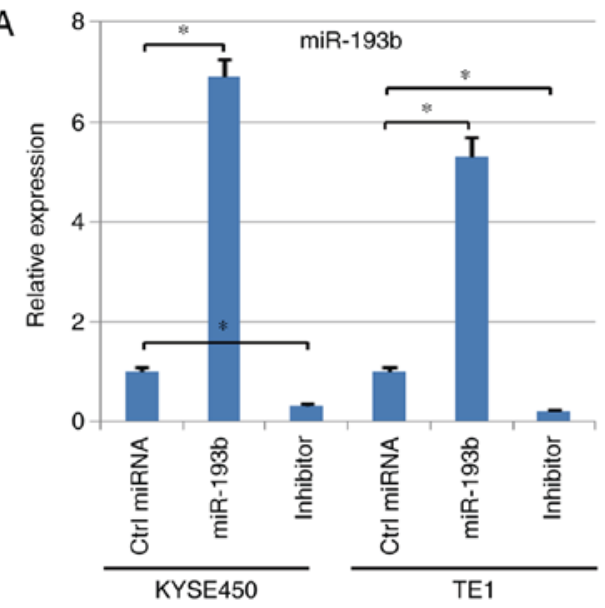

C

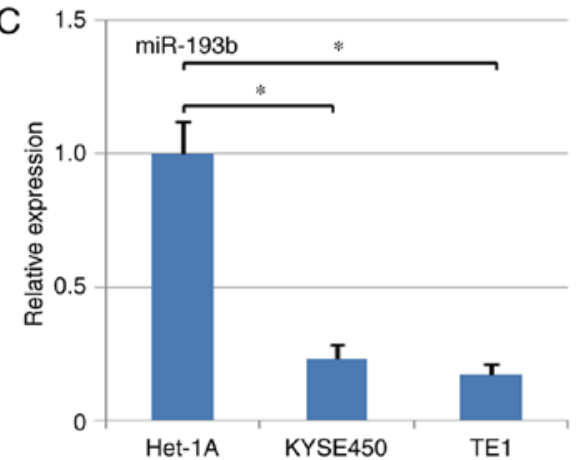

B

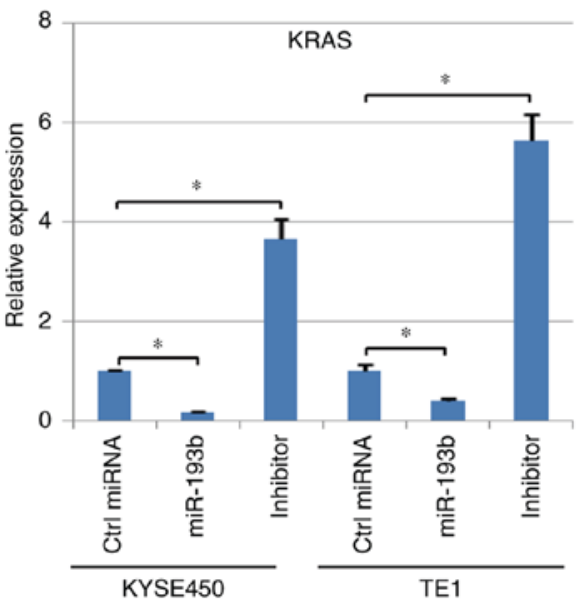

D

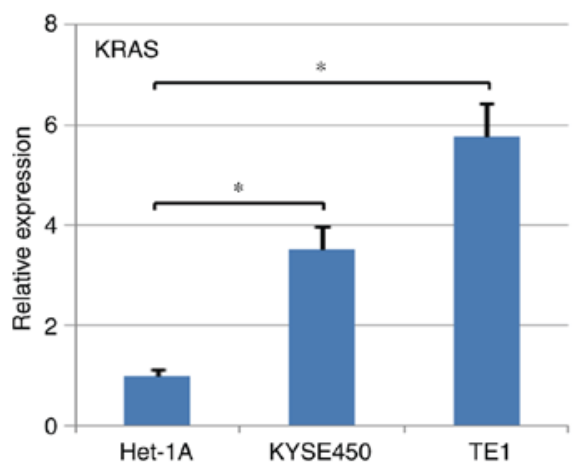

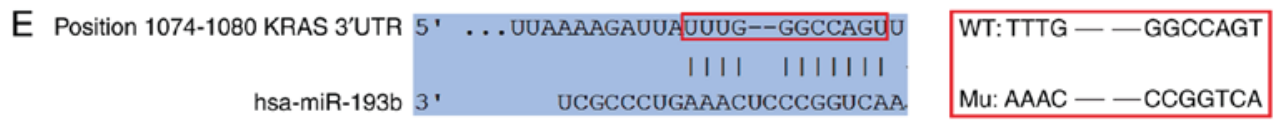
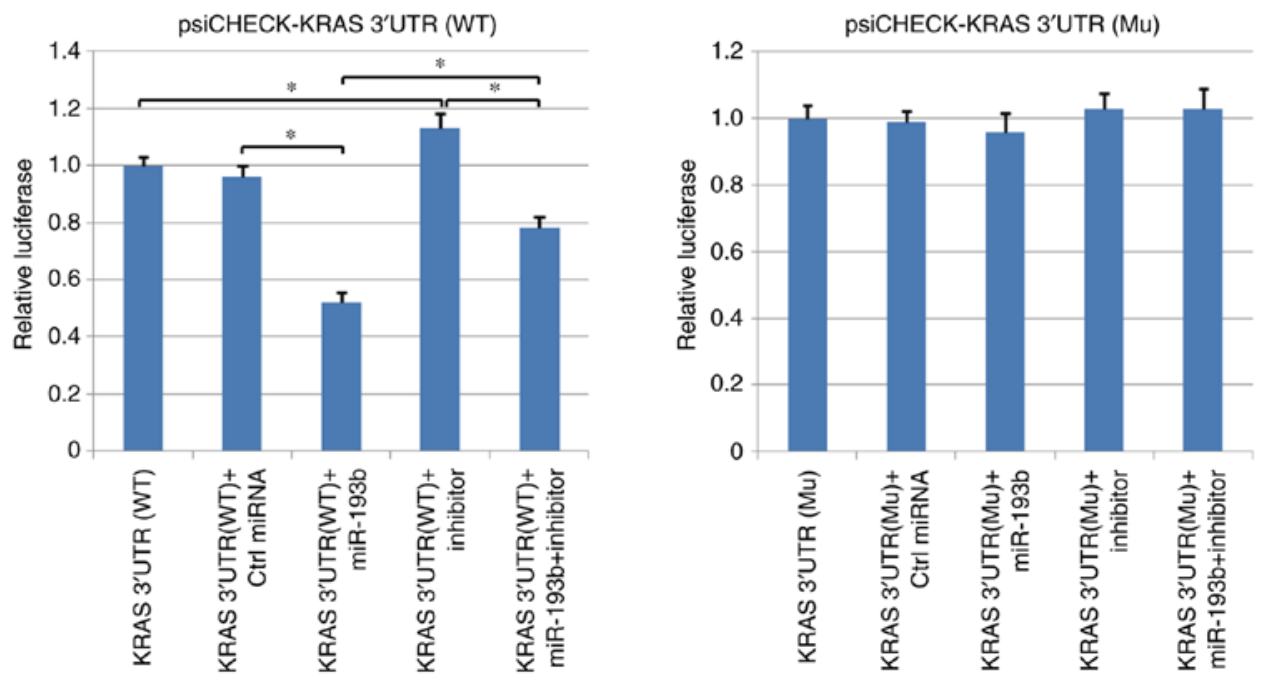

Figure 2. Expression of KRAS is regulated by miR-193b directly. (A and B) Effect of miR-193b overexpression/downregulation on the expression of KRAS in human esophageal squamous cell carcinoma cells. The level of (A) miR-193b and (B) KRAS in different groups was detected with qPCR. (C and D) Expression of KRAS and miR-193b in normal epithelial cell line, Het-1A, and KYSE450 and TE1 cell lines. (C) miR-193b and (D) KRAS expression was evaluated using qPCR. Their expression in Het-1A cells was compared to KYSE450 and TE1 cells. Normal epithelial cells Het-1A exhibited higher expression of miR-193b and lower expression of KRAS. (E) The direct binding relationship between miR-193b and KRAS. The targeted modulation between KRAS and miR-193b was analyzed using a dual-luciferase system herein. ${ }^{*} \mathrm{P}<0.05$ between the two groups. miR-193b, microRNA-193b.

carcinoma cells, indicating the key role of miR-193b in the development and progression of human esophageal cancer.

However, the detailed mechanism concerning the regulatory function of miR-193b in human esophageal squamous cell carcinoma still remains to be fully elucidated. Why the development of esophageal squamous cell carcinoma is accompanied with downregulation of miR-193b still requires further investigation. Some studies indicated that the level of hypermethylation in the promoter of some miRNAs could be enhanced in some cancer cells, resulting in the downregulation of those miRNAs (27). Therefore, hypermethylation in the promoter of miRNAs, may play an important role in the 

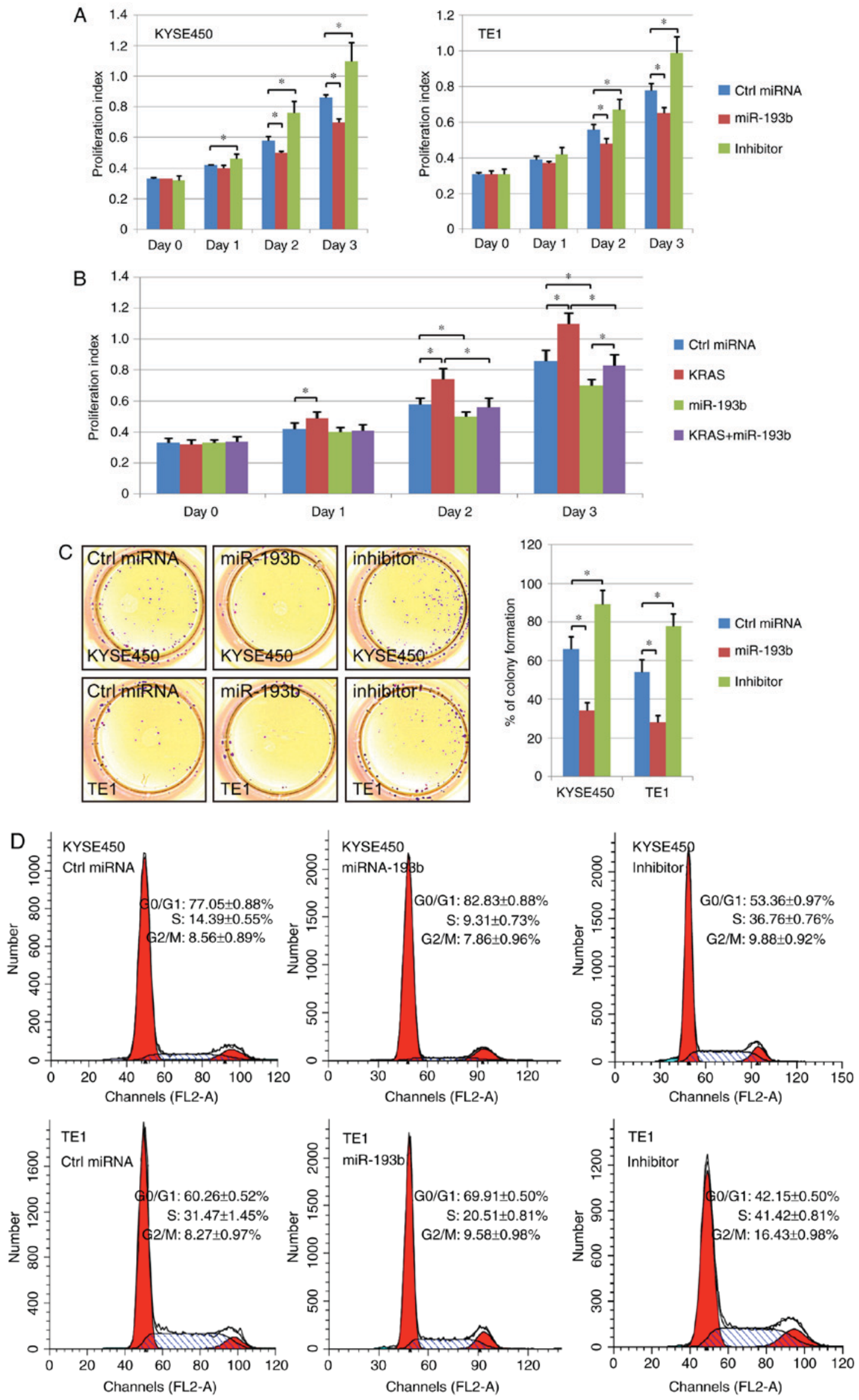

Figure 3. miR-193b overexpression and inhibitor affect the proliferation ability of human esophageal squamous cell carcinoma cells. The effect of miR-193b overexpression/inhibitor or KRAS on cell proliferation was detected with (A and B) CCK-8 assays, (C) colony formation assay and (D) cell cycle assay, respectively. ${ }^{*} \mathrm{P}<0.05$ between the two groups. miR-193b, microRNA-193b. 

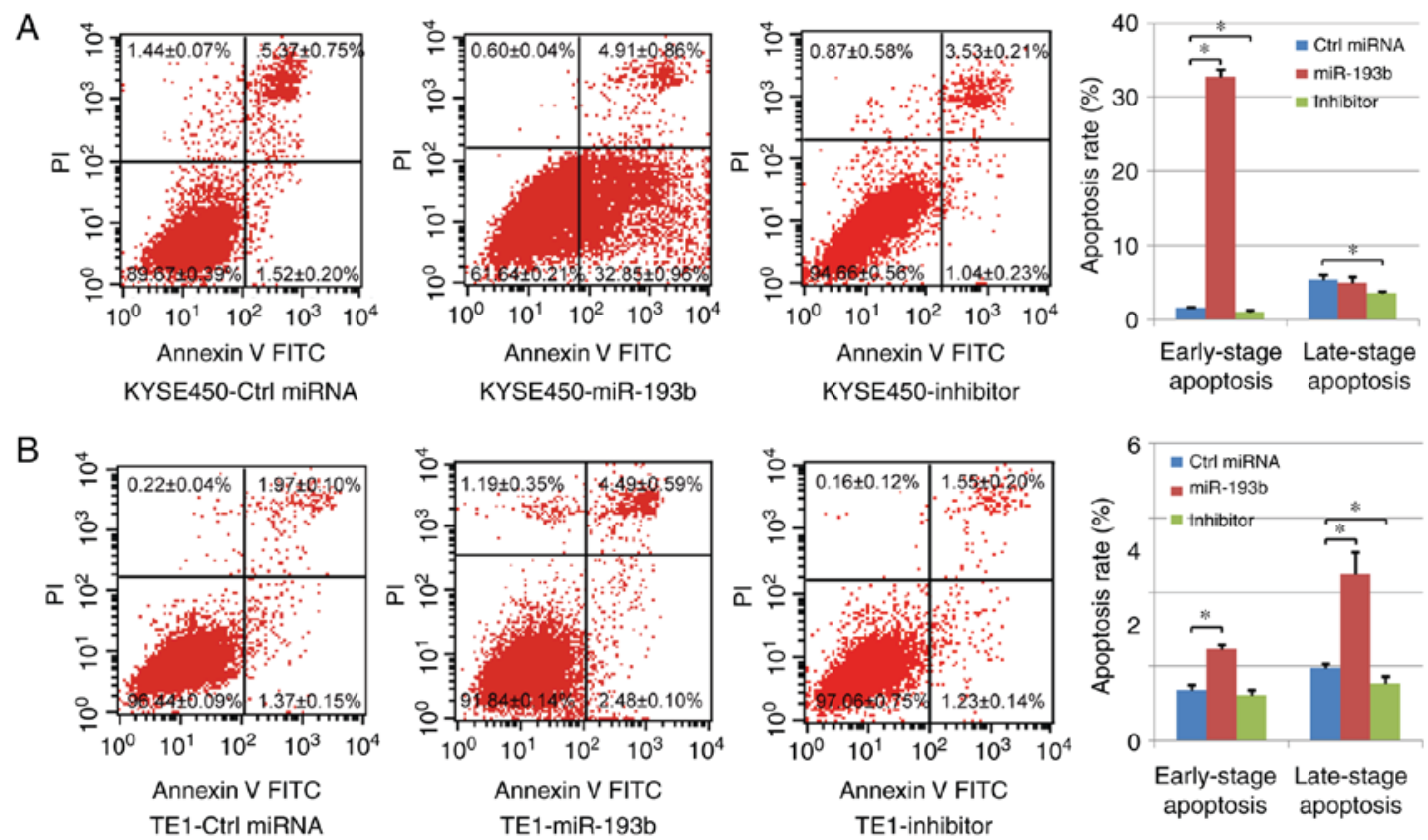

Figure 4. Effect of miR-193b on cell apoptosis. (A and B) Cell apoptosis was analyzed using Annexin V-FITC/PI staining in (A) KYSE450 cells and (B) TE1 cells. The summary of the apoptotic rate (\%) of early-stage (lower right quadrants) and late-stage (upper right quadrants) apoptotic cells. ${ }^{*} \mathrm{P}<0.05$ between the two groups. miR-193b, microRNA-193b.
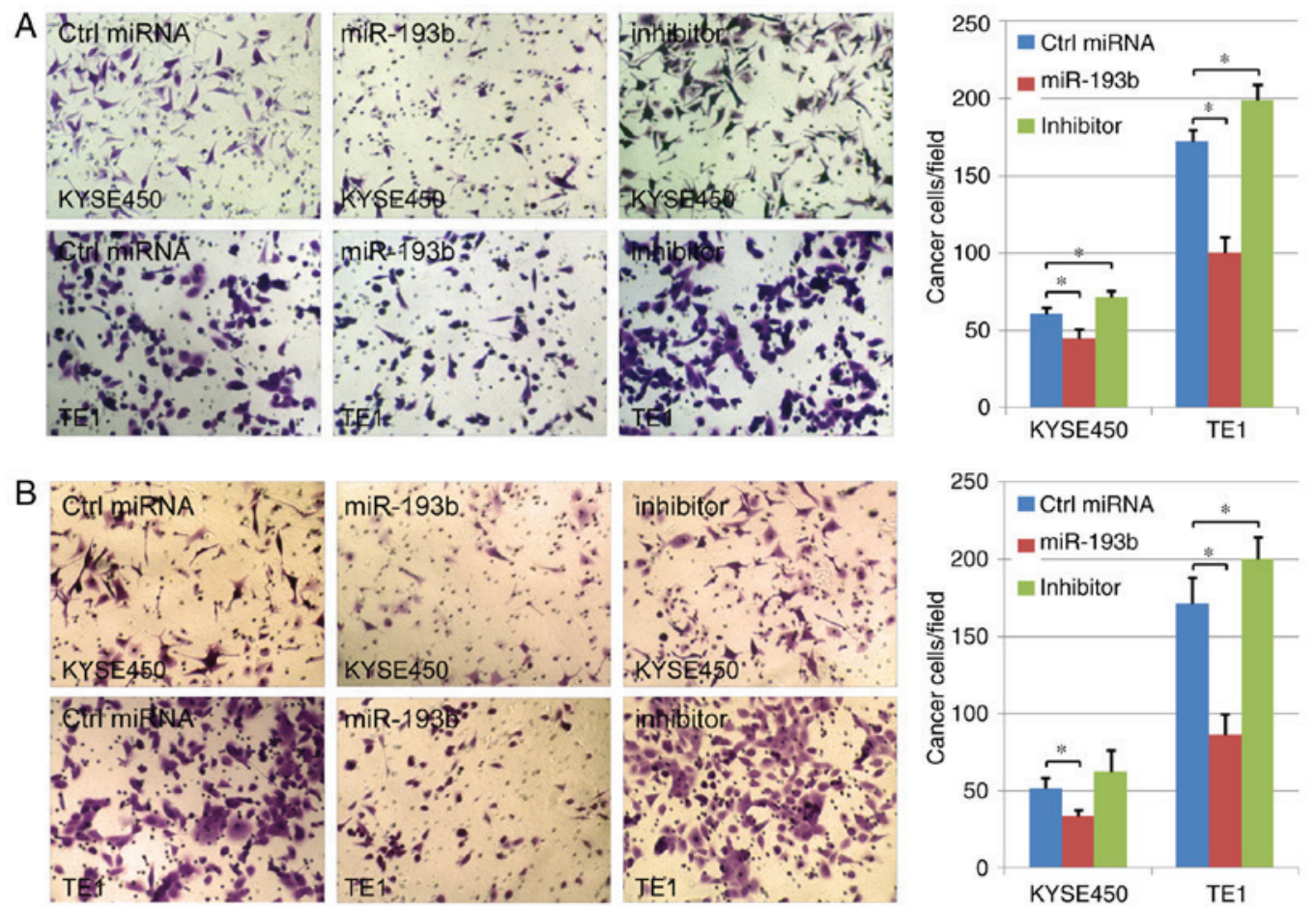

Figure 5. Cell migration and invasion assays. (A and B) Effect of miR-193b on the (A) migration and (B) invasion abilities of human esophageal squamous cell carcinoma cells. ${ }^{*} \mathrm{P}<0.05$ between the two groups. miR-193b, microRNA-193b.

regulation of miRNAs and in the development and progression of cancers. Nevertheless, whether such a hypermethylation mechanism is still applicable for the regulation of miR-193b still requires further investigation. Recently, research revealed that certain microenvironments induce the downregulation of miR-193b. In a previous study, the direct interaction between cancer cells and mesothelial cells, which covered the surface of the omentum, could decrease the expression level of miR-193b via DNA methyltransferase 1, providing another possible mechanism for the regulation of miR-193b in cancer progression (28).

Moreover, researchers have revealed other genes which could be regulated by miR-193b directly. For example, it was revealed that miR-193b was significantly downregulated in 
human Ewing sarcoma, and ErbB4 was further identified as a target of miR-193b. The overexpression of miR-193b significantly inhibited the expression of ErbB4 and the restoration of ErbB4 expression could reverse the inhibitory function of miR-193b on cancer cell proliferation as well as metastasis (29). Therefore, miR-193b can regulate the viability of cancer cells through different pathways. Thus, it may be useful to analyze different signaling pathways associated with miR-193b. A better understanding of the regulatory mechanism of miR-193b in different cancers and which gene plays the most important role during the process of miR-193b regulation, can help us develop more reliable and effective methods to treat patients with cancer.

In present study, our results revealed that miR-193b overexpression could decrease the viability of human esophageal cancer cell lines, and enhance cell apoptosis. However, slight differences were observed between the different cell lines. For example, miR-193b overexpression led to an increase in the percentage of both early-stage apoptotic cells and late-stage apoptotic cells in TE1 cells, while, overexpression of miR-193b only significantly increased the percentage of early-stage apoptotic cells in KYSE450 cells, and no obvious change was observed in late-stage apoptotic cells. It is possible that cancer cells with a high expression level of miR-193b or a low expression level of KRAS exhibit some resistance to miR-193b overexpression. Nevertheless, the application of more cell lines is required to confirm such a hypothesis and detailed mechanism.

In conclusion, the present study revealed that KRAS can be targeted by miR-193b in human esophageal squamous cell carcinoma, and miR-193b negatively regulated the expression of KRAS via binding to the 3'UTR of KRAS directly. miR-193b overexpression increased the level of apoptosis and inhibited cell proliferation ability as well as cell migration/invasion abilities in human esophageal squamous cell carcinoma cell lines via suppression of the expression of KRAS. Collectively, our results indicated that miR-193b holds promise as a novel diagnosis marker in human esophageal squamous cell carcinoma, as well as a target for gene therapy.

\section{Acknowledgements}

Not applicable.

\section{Funding}

The present study was supported by the National Natural Science Foundation of China (nos. 81570507, 81670474, 8130 2160)

\section{Availability of data and materials}

The analyzed datasets generated during the present study are available from the corresponding author on reasonable request.

\section{Authors' contributions}

PL, SG and SZhang conceived and designed the experiments. MK, SG, and YL performed the experiments. MK and SZhu analyzed the data. MK and SZhu contributed the reagents/materials/analysis tools. MK and SZhang wrote the paper. PL and SZhang supervised the experiments. All authors read and approved the manuscript and agree to be accountable for all aspects of the research in ensuring that the accuracy or integrity of any part of the work are appropriately investigated and resolved.

\section{Ethics approval and consent to participate}

This study was approved by the Committee on the Ethics of Animal Experiments and Human Subject Research of Capital Medical University (Beijing, China). All of the volunteers who donated cancer tissues and paracancerous tissues had provided written informed consent. The Ethics Committees of Capital Medical University had reviewed and approved this consent procedure.

\section{Patient consent for publication}

Not applicable.

\section{Competing interests}

The authors declare that they have no competing interests.

\section{References}

1. Jemal A, Bray F, Center MM, Ferlay J, Ward E and Forman D: Global cancer statistics. CA Cancer J Clin 61: 69-90, 2011.

2. Torre LA, Bray F, Siegel RL, Ferlay J,Lortet-Tieulent J and Jemal A: Global cancer statistics, 2012. CA Cancer J Clin 65: 87-108, 2015.

3. Napier KJ, Scheerer M and Misra S: Esophageal cancer: A Review of epidemiology, pathogenesis, staging workup and treatment modalities. World J Gastrointest Oncol 6: 112-120, 2014.

4. Wen SW, Zhang YF, Li Y,Liu ZX, Lv HL, Li ZH, Xu YZ, Zhu YG and Tian ZQ: Characterization and effects of miR-21 expression in esophageal cancer. Genet Mol Res 14: 8810-8818, 2015.

5. Gabra MM and Salmena L: microRNAs and acute myeloid leukemia chemoresistance: A mechanistic overview. Front Oncol 7: 255, 2017.

6. Cheng L, Zhan B, Luo P and Wang B: miRNA375 regulates the cell survival and apoptosis of human nonsmall cell carcinoma by targeting HER2. Mol Med Rep 15: 1387-1392, 2017.

7. Poltronieri P: Editorial: Overview on microRNAs in cancer development and virus infection. Microrna 5: 80-82, 2016.

8. Tutar L, Tutar E and Tutar Y: MicroRNAs and cancer; an overview. Curr Pharm Biotechnol 15: 430-437, 2014.

9. Rauhala HE, Jalava SE, Isotalo J, Bracken H, Lehmusvaara S, Tammela TL, Oja H and Visakorpi T: miR-193b is an epigenetically regulated putative tumor suppressor in prostate cancer. Int J Cancer 127: 1363-1372, 2010.

10. Chen J, Feilotter HE, Paré GC, Zhang X, Pemberton JG, Garady C, Lai D, Yang X and Tron VA: MicroRNA-193b represses cell proliferation and regulates cyclin D1 in melanoma. Am J Pathol 176: 2520-2529, 2010.

11. Li XF, Yan PJ and Shao ZM: Downregulation of miR-193b contributes to enhance urokinase-type plasminogen activator (uPA) expression and tumor progression and invasion in human breast cancer. Oncogene 28: 3937-3948, 2009.

12. Hummel R, Sie C, Watson DI, Wang T, Ansar A, Michael MZ, Van der Hoek M, Haier J and Hussey DJ: MicroRNA signatures in chemotherapy resistant esophageal cancer cell lines. World J Gastroenterol 20: 14904-14912, 2014.

13. Nyhan MJ, O'Donovan TR, Boersma AW, Wiemer EA and McKenna SL: MiR-193b promotes autophagy and non-apoptotic cell death in oesophageal cancer cells. BMC Cancer 16: 101, 2016.

14. Gupta B and Kumar N: Worldwide incidence, mortality and time trends for cancer of the oesophagus. Eur J Cancer Prev 26: 107-118, 2017.

15. Petty RD, Dahle-Smith A, Stevenson DAJ, Osborne A, Massie D, Clark C, Murray GI, Dutton SJ, Roberts C, Chong IY, et al: Gefitinib and EGFR gene copy number aberrations in esophageal cancer. J Clin Oncol 35: 2279-2287, 2017. 
16. Zhang R, Li Y, Chen Y, Liu X, Wang Z, Sun H, Zheng Y, Ding Z, Lan L, Li M, et al: Clinical implications of the concentration and EGFR/KRAS mutations of plasma cell free DNA of patients with lung cancer and esophageal cancer. Zhonghua Yi Xue Za Zhi 95: 3839-3842, 2015 (In Chinese).

17. Liu P, Feng Y, Dong D, Liu X, Chen Y, Wang Y and Zhou Y: Enhanced renoprotective efect of IGF-1 modifed human umbilical cord-derived mesenchymal stem cells on gentamicin-induced acute kidney injury. Sci Rep 6: 20287, 2016.

18. Liu P, Cai J, Dong D, Chen Y, Liu X, Wang Y and Zhou Y: Effects of SOX2 on proliferation, migration and adhesion of human dental pulp stem cells. PLoS One 10: e0141346, 2015.

19. Liu P, Chen S, Li X, Qin L, Huang K, Wang L, Huang W, Li S, Jia B, Zhong M, et al: Low immunogenicity of neural progenitor cells differentiated from induced pluripotent stem cells derived from less immunogenic somatic cells. PLoS One 8: e69617, 2013

20. Liu Y, Wu T, Song J, Chen X, Zhang Y and Wan Y: A mutant screening method by critical annealing temperature-PCR for site-directed mutagenesis. BMC Biotechnol 13: 21, 2013.

21. Liu P, Zhang Y, Chen S, Cai J and Pei D: Application of iPS cells in dental bioengineering and beyond. Stem Cell Rev 10: 663-670, 2014.

22. Yao Q, Pei Y, Zhang X and Xie B: microRNA-96 acts as a tumor suppressor gene in human osteosarcoma via target regulation of EZRIN. Life Sci 203: 1-11, 2018.

23. Chen K, Liu MX, Mak CS, Yung MM, Leung TH, Xu D, Ngu SF, Chan KK, Yang H,Ngan HY and Chan DW: Methylation-associated silencing of miR-193a-3p promotes ovarian cancer aggressiveness by targeting GRB7 and MAPK/ERK pathways. Theranostics 8 : 423-436, 2018.
24. Jin X, Sun Y, Yang H, Li J, Yu S, Chang X, Lu Z and Chen J: Deregulation of the MiR-193b-KRAS axis contributes to impaired cell growth in pancreatic cancer. PLoS One 10: e0125515, 2015.

25. Gastaldi C, Bertero T, Xu N, Lebrigand K, Fourre S, Popa A, Cardot-Leccia N, Meneguzzi G, Sonkoly E, et al: miR-193b/365a cluster controls progression of epidermal squamous cell carcinoma. Carcinogenesis 35: 1110-1120, 2014.

26. Li J, Kong F, Wu K, Song K, He J and Sun W: miR-193b directly targets STMN1 and uPA genes and suppresses tumor growth and metastasis in pancreatic cancer. Mol Med Rep 10: 2613-2620, 2014

27. Lü L, Liu T, Gao J, Zeng H, Chen J, Gu X and Mei Z: Aberrant methylation of microRNA-193b in human Barrett's esophagus and esophageal adenocarcinoma. Mol Med Rep 14: 283-288, 2016.

28. Mitra AK, Chiang CY, Tiwari P, Tomar S, Watters KM, Peter ME and Lengyel E: Microenvironment-induced downregulation of miR-193b drives ovarian cancer metastasis. Oncogene 34: 5923-5932, 2015.

29. Moore C, Parrish JK and Jedlicka P: MiR-193b, downregulated in Ewing Sarcoma, targets the ErbB4 oncogene to inhibit anchorage-independent growth. PLoS One 12: e0178028, 2017. 